\title{
Nature (genes), nurture (epigenetics), and brain development
}

Volume 6 Issue 2 - 2017

\section{Introduction}

It is not always clear what mechanisms cause a child to have cognitive or behavioral dysfunction to inherit a developmental disorder though siblings, even twins, may not. Developmental neuroscience literature has previously placed an emphasis on the role of genetics on development. However, current neuroscience literature suggests that the focus should be shifted toward the effects of the environment on epigenetics, or, the study of heritable changes in gene function that occur without a primary change in the DNA sequence. Some developmental disorders such as reading disorders have are thought to be highly genetically driven ${ }^{1,2}$ However, it is likely that the combination of genes and environmental factors that activate them is likely what causes many developmental problems.

Behavioral geneticists commonly claim that DNA accounts for 30 to 50 percent of our behaviors, ${ }^{3}$ which leaves 50 to 70 percent explained by environment. It is now clear that there is a dynamic interplay between genes and environmental influences. Epigenetics may help to explain causes of brain dysfunction via acting as a conduit through which environmental factors elicit potentially lifelong biological changes. For example, epigenetic factors may help explain how identical twins often don't develop the same cognitive or psychiatric disorders and family variability in functioning. Epigenetics is thought to play a role in central nervous system (CNS) development, cognitive functioning, addiction, and psychopathology $\mathrm{y}^{4}$ and has been implicated in the development of post-traumatic stress disorder, Alzheimer's disease, Schizophrenia, and neurodevelopmental disorders including Autism Spectrum Disorder, and Rett Syndrome. ${ }^{5,6}$

\section{Epigenetic mechanisms}

Numerous empirical studies have shown the potential long-term emotional, behavioral, and cognitive effects of psychological trauma, abuse, neglect, and various other environmental variables. It is thought that the stress experience of in these conditions may cause epigenetic changes and thus long-term change. There are thought to be over 40 types of epigenetic modifications. ${ }^{6}$ One such modification includes changes in histone and this is common to individuals with autism spectrum disorder. Environmental factors are thought to turn various functional genetic switches "on" or "off". Genes can be activated or deactivated by a host of environmental factors such as stress and nutrition. Though the experience of eustress (i.e., neutral or positive amount of psychological stress), for example, is a normal part of development, stressors can cause toxic stress or trigger maladaptive gene expression. Epigenetic changes are associated with brain dysfunction including PTSD, Bipolar Disorder, Major Depression, addiction, and Epilepsy ${ }^{4,5}$ and other common disorders. The association of early childhood experience, behavioral symptoms, and epigenetic alterations has been observed in humans. Researchers have observed striking differences in epigenetic profiles when comparing the brains of people who experienced childhood abuse and committed

\author{
Robert Perna, Lindsey Harik \\ Clinical neuropsychologist, TIRR Memorial Hermann, USA
}

Correspondence: Robert Perna, RN, Ph.D. Clinical neuropsychologist, TIRR Memorial Hermann, Texas Medical Center Houston, 2450 Holcombe Blvd \#I, Houston, TX 7702I, USA, Tel 706-750-2572, Email dr.perna@juno.com

Received: January 31, 2017 | Published: February 07, 2017

suicide later in life with the brains of people who did not experience such childhood trauma and later committed suicide. ${ }^{7}$

The prenatal and postnatal periods are characterized by rapid changes in neuronal organization providing a critical window of opportunity during which environmental experiences can lead to longterm influences on brain and behavior. Some literature ${ }^{8}$ suggests the involvement of epigenetic regulatory mechanisms in the pathogenesis of autism spectrum disorders (ASD) has been suggested due to the recognition of occurrence of ASD in patients with neurogenetic conditions that arise from disorders involving epigenetic regulatory factors (e.g., Fragile X syndrome, Rett syndrome, microdeletion disorders). The role of epigenetic modification in sustaining the effects of environmental experience has also been demonstrated in the context of postnatal mother-infant interactions. Individual variations in maternal care during the immediate postpartum period in rats are associated with changes in offspring hypothalamic-pituitary-adrenal (HPA) activity, neuroendocrine systems involved in reproduction, and hippocampal plasticity ${ }^{9}$

Unlike the DNA sequence, epigenetic processes are dynamic and not fixed, although some can persist for up to several years or an entire lifetime. Further, they are strongly influenced by the environment and by exposure to external factors like diet, living conditions, exercise, stress, chemicals, drugs, and toxins. Both positive and negative factors can modulate the epigenome. For instance, positive factors such as enriched living conditions, like social interactions, physical activity, and changing surroundings can promote beneficial epigenetic marks, while severe stress or agricultural chemicals can permanently alter some genes ${ }^{10}$ in a harmful manner. Furthermore, traumatic events and severe chronic stress in early life can alter the epigenome in a persistent and sometimes heritable fashion. ${ }^{11,12}$

\section{Epigenetics and memory}

Recent work in the field of neurobiology has showed that several enzymes that modify DNA or histone proteins are essential elements of signaling pathways. These pathways are thought to allow proper 
neuronal signaling for learning and memory. ${ }^{13}$ It is thought that long-term memory requires that epigenetic processes induce lasting changes in gene expression in brain cells. Mice with dysfunctions in any of the epigenetic components that contribute to these changes can have impaired long-term memory. ${ }^{13,14}$ Because epigenetic modulation is a necessary aspect of memory processes, there are scientists that are currently exploring the possibility of using epigenetic therapies to treat disorders that involve memory and other cognitive disorders. ${ }^{15,16}$

Epigenetic research is helping to further our understanding of developmental disorders and acquired brain dysfunction and will likely lead to innovative treatments for a variety of disorders. One potential benefit from this line of research is the ability to determine which environmental factors promote or diminish gene expression in varying disorders. Perhaps neuroepigeneticallyinformed environmental modification will decrease the incidence of developmental disorders in individuals with known genetic precursors. Epigenetics may one day be able to alleviate otherwise potential long-term symptoms. Clinicians may benefit from gaining an understanding of common neuroepigenetic mechanisms and potential triggers in their population(s) of interest.

\section{Acknowledgments}

None.

\section{Conflicts of interest}

Author declares there are no conflicts of interest.

\section{Funding}

None.

\section{References}

1. Saudino. Behavioral genetics and child temperament. J Dev Behav Pediatr. 2005;26(3):214-223.

2. National Institute of Health. Neuroepigenetic promise: A better understanding of behavior, learning, and mental disorders. 2015.

3. Sweatt JD. The emerging field of neuroepigenetics. Neuron. 2013;80(3):624-632.
4. Silvia P. Molecular Genetics of Dyslexia. In: eLS. Chichester, John Wiley \& Sons Ltd, USA. 2009.

5. Tsankova N, Renthal W, Kumar A, et al. Epigenetic regulation in psychiatric disorders. Nat Rev Neurosci. 2007;8(5):355-367.

6. Fisher SE. DeFries JC. Developmental Dyslexia: Genetic Dissection of a Complex Cognitive Trait. Nat Rev Neurosci. 2002;3(10):767-780.

7. McGowan PO, Sasaki A, D'Alessio AC, et al. Epigenetic regulation of the glucocorticoid receptor in human brain associates with childhood abuse. Nat Neurosci. 2009 12(3):342-348.

8. Zhao X, Pak C, Smrt RD, et al. Epigenetics and Neural developmental disorders. Epigenetics. 2007;2(2):126-138

9. Meaney MJ. Maternal care, gene expression, and the transmission of individual differences in stress reactivity across generations. Annu Rev Neurosci. 2001;24: 1161-1192.

10. Portela A, Esteller M. Epigenetic modifications and human disease. Nat Biotechnol. 2010;28(10):1057-1068.

11. Franklin TB, Mansuy IM. Epigenetic inheritance in mammals: Evidence for the impact of adverse environmental effects. Neurobiol Dis . 2010;39(1):61-65.

12. Franklin TB, Russig H, Weiss IC, et al. Epigenetic transmission of the impact of early stress across generations. Biol Psychiatry. 2010;68(5):408-415.

13. Alarcón JM, Malleret G, Touzani K, et al. Chromatin acetylation, memory, and LTP are impaired in $\mathrm{CBP}+/$ - mice: A model for the cognitive deficit in Rubinstein-Taybi syndrome and its amelioration. Neuron. 2004;42(6):947-959.

14. Korzus E, Rosenfeld MG, Mayford M. CBP histone acetyltransferase activity is a critical component of memory consolidation. Neuron. 2004;42(6):961-972.

15. Urdinguio RG, Sanchez-Mut JV, Esteller M. Epigenetic mechanisms in neurological diseases: genes, syndromes, and therapies. Lancet Neurol. 2009;8(11):1056-1072.

16. Gräff J, Koshibu K, Jouvenceau A, et al. Protein phosphatase 1-dependent transcriptional programs for long-term memory and plasticity. Learn Mem. 2010;17(7):355-363. 